\title{
Targeting colorectal cancer cell metabolism through development of cisplatin and metformin nano-cubosomes
}

\author{
Mona M. Saber ${ }^{1,2^{*}}$, Abdulaziz M. Al-mahallawi ${ }^{3}$, Noha N. Nassar ${ }^{1}$, Björn Stork ${ }^{2^{*}}$ (iD and Samia A. Shouman ${ }^{4}$
}

\begin{abstract}
Background: Colorectal cancer (CRC) remains a leading cause of death worldwide. Utilizing cisplatin in CRC is correlated with severe adverse effects and drug-resistance. Combined anticancer drug-treatment, along with, their enhanced delivery, can effectively kill cancer through multiple pathways. Nano-cubosomes are emerging as nanocarriers for anticancer therapies, hence, we constructed nano-cubosomes bearing cisplatin and cisplatinmetformin combination for investigation on HCT-116 cells.

Methods: Nano-cubosomes bearing either cisplatin alone or cisplatin-metformin combination were formulated using emulsification technique. The loaded nano-cubosomes were characterized in vitro and the optimized formulation was selected. Their cytotoxic effects were investigated by Sulphorhodamine-B (SRB) assay. The AMPK mTOR metabolic pathway as well as the Akt/mTOR pathway were analyzed using ELISA technique. Colorimetry was used in NADPH oxidase, LDH and caspase-3 activity determination.

Results: nano-cubosomal formulations exhibited superior cytotoxic effect compared to unformulated cisplatin. This cytotoxic effect was profound upon incorporation of metformin, an indirect mTOR inhibitor, in cisplatin nanocubosomes. The induced CRC cell apoptosis was through inhibition of several metabolic pathways, namely, AMPK mTOR and Akt/mTOR. Drug-loaded nano-cubosomes ensued depletion in glucose and energy levels that led to AMPK activation and thus mTOR inhibition. mTOR was additionally inhibited via suppression of p-Akt (Ser473) levels after nano-cubosomal treatment. Moreover, drug-loaded nano-cubosomes produced a notable escalation in ROS levels, evident as an increase in NADPH oxidase, inhibition of LDH and a consequential upsurge in caspase-3.
\end{abstract}

Conclusion: These results demonstrated the influence exerted by cisplatin-loaded nano-cubosomes on CRC cell survival and enhancement of their cytotoxicity upon metformin addition.

Keywords: AMPK, Cisplatin, Metformin, mTOR, Nano-cubosomes

\section{Background}

Colorectal cancer (CRC) is one of the most common causes of cancer-related death worldwide, with over a million newly diagnosed cases per year. Although, cisplatin is designated as a golden chemotherapeutic agent in solid tumor treatment, in CRC, therapy is accompanied by dose-limiting adverse effects, resistance and

\footnotetext{
* Correspondence: mona.magdy@pharma.cu.edu.eg; mona.saber@uniduesseldorf.de; bjoern.stork@uni-duesseldorf.de

'Department of Pharmacology and Toxicology, Faculty of Pharmacy, Cairo University, Kasr El-Aini St, Cairo 11562, Egypt

${ }^{2}$ Institute of Molecular Medicine I, Medical Faculty, Heinrich-Heine-University, Universitätsstr. 1, Building 23.12, 40225 Düsseldorf, Germany

Full list of author information is available at the end of the article
}

decreased effectiveness. Literature suggests that cisplatin effectiveness as a chemotherapeutic agent in CRC could be improved by combinatorial approaches [1].

Metformin received increased attention for its potential anti-tumorigenic effects which are independent of its blood glucose lowering action; notably, studies revealed lower CRC incidence and mortality with its use [2]. On CRC cell lines, it inhibited cell growth and synergistically increased apoptosis when combined with other chemotherapy drugs [3]. One proposed antitumor mechanism of metformin is reprogramming of cancer cell metabolism that results in forceful effects on gene expression,

(c) The Author(s). 2018 Open Access This article is distributed under the terms of the Creative Commons Attribution 4.0 International License (http://creativecommons.org/licenses/by/4.0/), which permits unrestricted use, distribution, and reproduction in any medium, provided you give appropriate credit to the original author(s) and the source, provide a link to the Creative Commons license, and indicate if changes were made. The Creative Commons Public Domain Dedication waiver (http://creativecommons.org/publicdomain/zero/1.0/) applies to the data made available in this article, unless otherwise stated. 
cell differentiation, proliferation and tumor microenvironment [4].

Advances in chemotherapy treatment of CRC are limited to the currently available selection of licensed drugs, most of which have been used for many years. The slow progress in disease treatment resulted in an increased need for a new approach like nano-systems to overcome problems of drug delivery. Nano-systems use in cancer offer the possibility to enhance drug efficacy with minimal side effects. Despite the remarkable development of different nanoparticle types for various purposes, relatively little is known about the cellular interactions and toxicity of monoolein-based nano-cubosomes. Several nanoparticle preparations containing cisplatin were formulated, including liposomes, gold nanoparticles and dendrimers, however, the anticipated improvement in efficacy was not observed [5]. To this end, the current study aimed to formulate nano-cubosomes (self-assembled cubic liquid crystalline nanoparticles) incorporating cisplatin and metformin in an attempt to increase efficacy in HCT-116 CRC cells, as well as, investigating the possible effect on tumorigenesis-associated metabolism.

\section{Methods}

\section{Drugs}

Cisplatin and metformin were obtained from Mylan (Virginia, USA) and CID Co. (Cairo, Egypt). The drugs were freshly dissolved in saline and all experiments carried out protected from light.

\section{Chemicals}

Roswell Park Memorial Institute-1640 (RPMI-1640), glyceryl monooleate (GMO), polyvinyl alcohol (PVA), Pluronic-F127 and sulphorhodamine-B (SRB) were obtained from Sigma Chemical Co. (St. Louis, USA). All other chemicals and reagents used were of analytical grade and used without further purification.

\section{Preparation of nano-cubosomal dispersions}

Nano-cubosomal dispersions with average particle size ranging from $120 \mathrm{~nm}$ to $150 \mathrm{~nm}$ and polydispersity index from 0.05 to 0.20 were prepared. The prepared nano-formulations exhibited zeta potential from $30 \mathrm{mV}$ to $-45 \mathrm{mV}$ indicating that they have sufficient charges that would inhibit their aggregation [6] (Fig.1). The nano-cubosomes were prepared by emulsification technique described by Morsi et al. [7] with slight modifications. For blank nano-cubosomes, GMO and Pluronic-F127 were melted at $70{ }^{\circ} \mathrm{C}$. The obtained molten solution was added drop-wise to $20 \mathrm{~mL}$ normal saline $\left(70{ }^{\circ} \mathrm{C}\right)$ containing 2.5\% PVA under mechanical stirring at $1500 \mathrm{rpm}$. Dispersions were maintained under stirring and cooled to room temperature to achieve homogenous state followed by probe sonication for $10 \mathrm{~min}$ (at 70\% amplitude) [8]. For the preparation of drug-loaded nano-cubosomes, the drugs (cisplatin or cisplatin-metformin combination) were added to the aqueous phase prior to addition of the molten hydrophobic phase. The final cisplatin concentration in the nano-cubosomal dispersion was $60 \mu \mathrm{M}$. Formulas were freshly prepared before each experiment. Transmission electron microscope (TEM) revealed that the prepared cubosomes are in the nano-size, which confirms the results of particle size measurement, and showed nanoparticles of cubical nanostructure (Fig.2).

\section{Cell culture}

Human CRC cell line, HCT-116 (ATCC ${ }^{\circ}$ CCL-247 $7^{\mathrm{mm}}$ ), was obtained from the American Type Culture Collection (Manassas, USA). At the Egyptian National Cancer Institute (Cairo, Egypt), it was maintained and grown in RPMI-1640 supplemented with $10 \%$ fetal bovine serum, $1.5 \mathrm{~g} / \mathrm{L}$ sodium bicarbonate, $2 \mathrm{mM} \mathrm{L}$ glutamine and $1 \%$ penicillin/streptomycin in $5 \% \mathrm{CO}_{2}$ at $37^{\circ} \mathrm{C}$.

\section{Cytotoxicity assay}

SRB assay was used to evaluate cytotoxicity. Exponentially growing cells were seeded in 96-well plates at an initial density of $5 \times 10^{3} /$ well. Nano-cubosomes were added after $24 \mathrm{~h}$ with various concentrations and incubated at $37{ }^{\circ} \mathrm{C}$ for $48 \mathrm{~h}$ to determine their $\mathrm{IC}_{50} \mathrm{~s}$ (the concentration of the drug required to produce $50 \%$ cell growth inhibition). Cells were fixed with trichloroacetic acid (10\%) for $1 \mathrm{~h}$ at $4{ }^{\circ} \mathrm{C}$, stained with SRB $(0.4 \%)$ for $30 \mathrm{~min}$., then washed four times with acetic acid (1\%) and air-dried. The dye was dissolved with $10 \mathrm{mM}$ Tris base ( $\mathrm{pH} 10.5)$ before measuring the optical density (O.D.) spectrophotome trically at $570 \mathrm{~nm}$ with the microplate reader (Tecan SunriseTM, Switzerland). Cell survival fraction was calculated as follows: survival fraction $=$ O.D. (treated cells)/O.D. (control cells). The $\mathrm{IC}_{50} \mathrm{~s}$ after $48 \mathrm{~h}$ treatment were calculated using sigmoidal dose-response curve-fitting models (Graphpad Prism Software, version 5.03, USA). The concentration of cisplatin nano-cubosomes that inhibited $30 \%$ of the cells was determined and used to prepare different nano-cubosomal formulations containing fixed $\mathrm{IC}_{30}$ of cisplatin and different metformin concentrations. This was done to identify the metformin concentration that will produce an $\mathrm{IC}_{50}$ when used with cisplatin. The concentrations used in all experiments were the $\mathrm{IC}_{30}$ of the cisplatin nano-cubosomes $(7 \mu \mathrm{M})$, cisplatin $(7 \mu \mathrm{M})$-metformin $(7 \mathrm{mM})$ nano-cubosomes and the same concentration of the single drugs as their respective controls. 


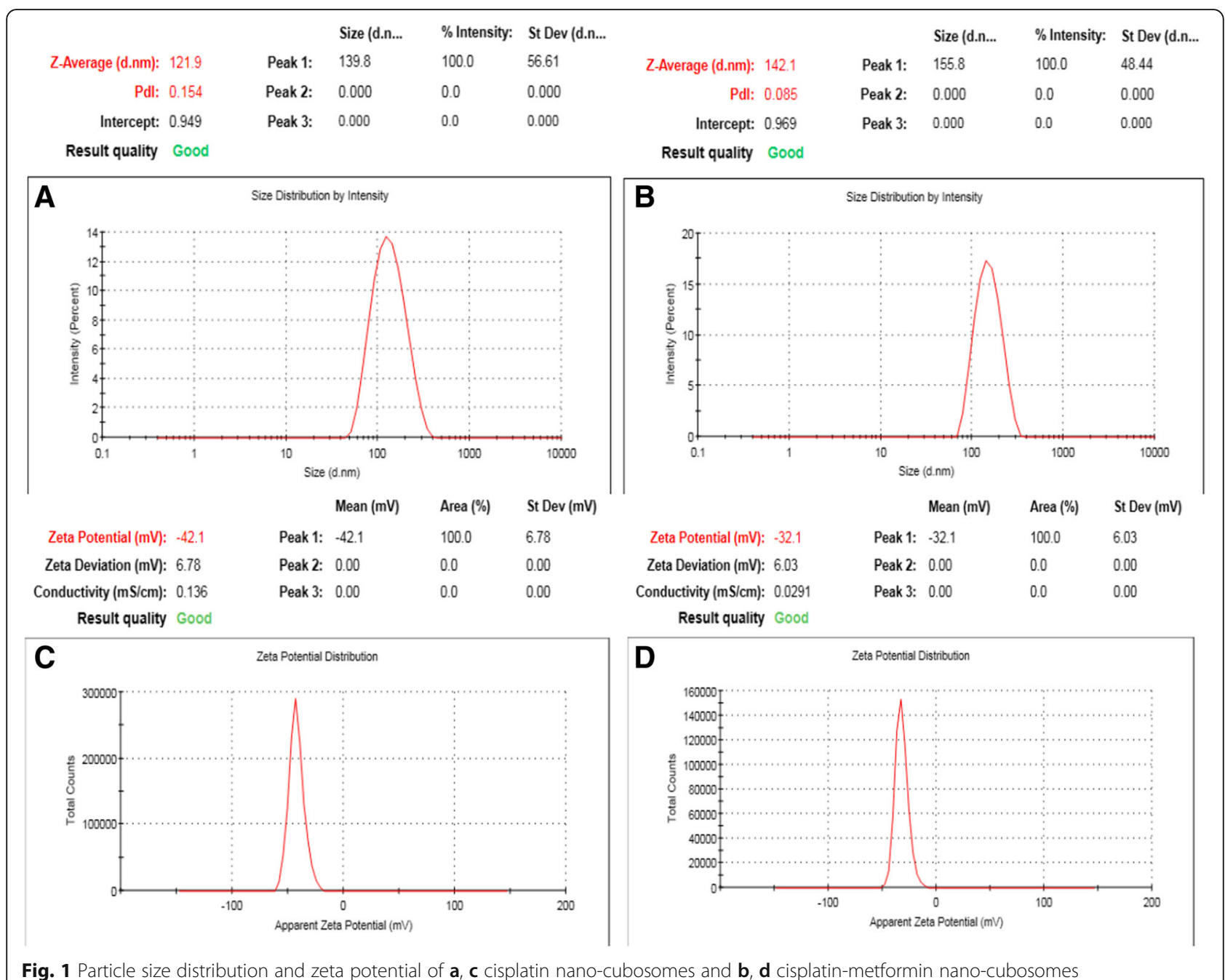

\section{Evaluation of drug interaction}

Concentration response curves of metformin and cisplatin alone in HCT-116 cells were first generated. The interaction between metformin and cisplatin in nano-cubosomes was then analyzed by calculating the combination index $(\mathrm{CI})$ using the following isobologram equation:

$$
\mathrm{CI}=\mathrm{d} 1 / \mathrm{D} 1+\mathrm{d} 2 / \mathrm{D} 2
$$

Where $\mathrm{d} 1$ and $\mathrm{d} 2$ are the respective concentrations of the drugs used in the combination required to produce a fixed level of inhibition while D1 and D2 are their concentrations able to produce alone the same magnitude of effect. If CI is less than 1 , the interaction between the two drugs is synergistic, while if $\mathrm{CI}=1$ or $>1$, the interaction is additive or antagonistic, respectively.

\section{Determination of cisplatin levels}

After treating HCT-116 cells with cisplatin, cisplatin nano-cubosomes and cisplatin-metformin nano-cubo somes for $48 \mathrm{~h}$, cells were collected, washed with PBS solution, lysed, centrifugated and supernatant separated to obtain a clear cell lysate. Cisplatin levels were determined according to the method developed by Golla and Ayres which is based on complexing cisplatin with o-phenylenediamine to give a green color [9]. The product was obtained at $\mathrm{pH} 6.2$, in $30 \mathrm{~min}$ at $90{ }^{\circ} \mathrm{C}$, giving a maximum absorbance at $705 \mathrm{~nm}$.

\section{Measurement of glucose, ATP and lactate levels}

After treatment of cells with desired concentrations of metformin, cisplatin, cisplatin nano-cubosomes and cisplatin-metformin nano-cubosomes for $48 \mathrm{~h}$, the medium was collected for glucose level analysis and cells were harvested and washed in PBS for ATP level measurement. Glucose level was detected using Spinreact glucose-TR kit (Santa Coloma, Spain) based on its oxidation to gluconic acid by glucose oxidase. The formed hydrogen peroxide, is detected by a chromogenic oxygen 

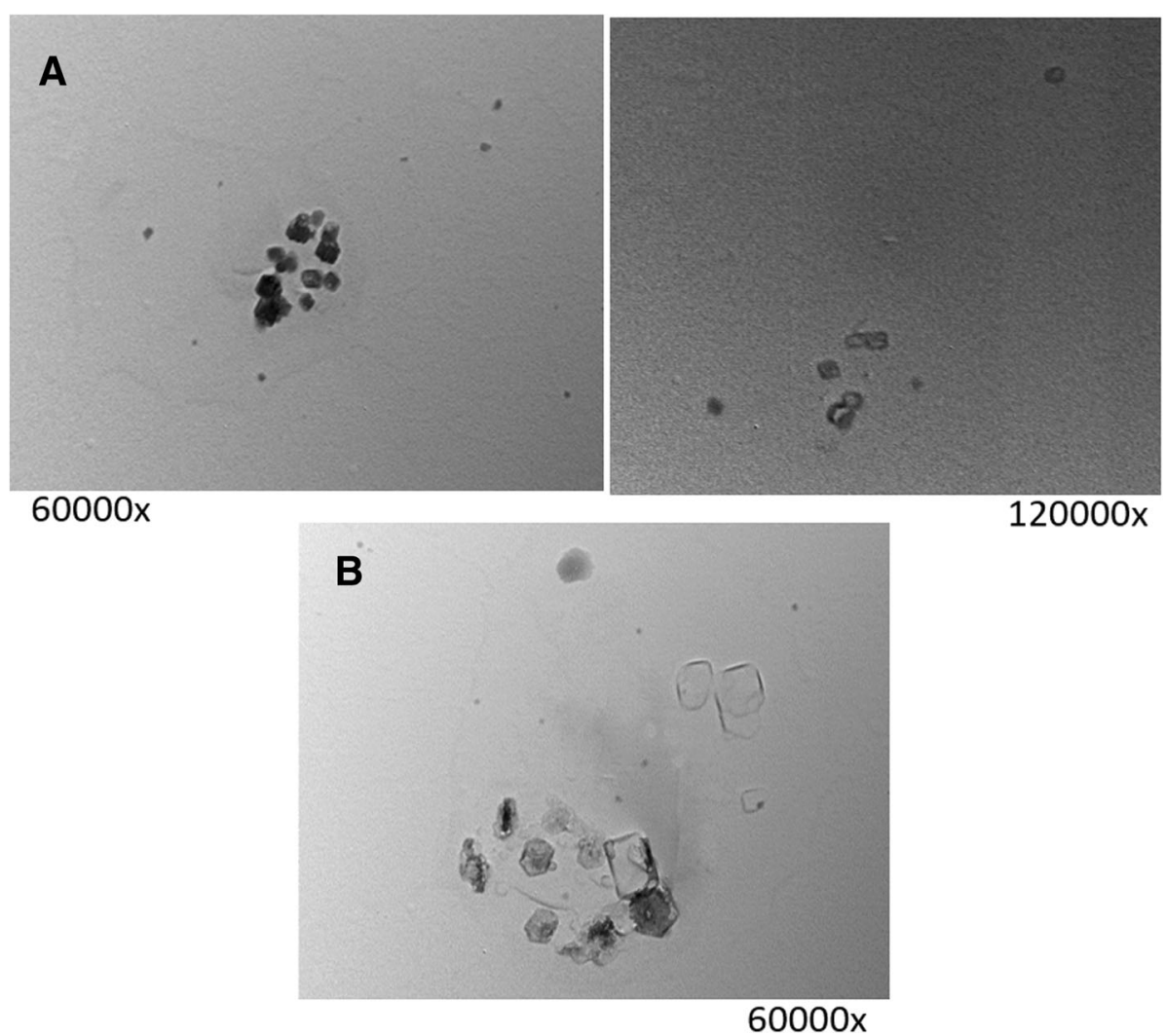

Fig. 2 Transmission electron micrographs of different nano-cubosomal dispersions; a cisplatin nano-cubosomes and $\mathbf{b}$ cisplatin-metformin nano-cubosomes

acceptor. The intensity of the color formed is measured at $505 \mathrm{~nm}$ and is proportional to glucose concentration which was calculated with reference to a glucose standard solution.

ATP levels were measured in the cell lysate using ATP colorimetric assay kit (BioVision, USA) which utilizes the phosphorylation of glycerol to generate a product that is quantified colorimetrically at $570 \mathrm{~nm}$.

Lactate levels in the cell culture medium were measured using L-lactate colorimetric assay kit (Abcam, U.K) where lactate is oxidized by lactate dehydrogenase to generate a product that interacts with a probe to produce a color that is measured at $450 \mathrm{~nm}$.

\section{Assessment of AMP/ATP ratio}

To further investigate the energy status of the HCT-116 cells, AMP/ATP ratios after $24 \mathrm{~h}$ and $48 \mathrm{~h}$ were measured. The cells were treated with metformin, cisplatin, cisplatin nano-cubosomes and cisplatin-metformin nano-cubosomes, then harvested and washed with PBS. The AMP/ATP ratios were determined using ATP/ADP/AMP assay kit (Biomedical Research Service, NY, USA) according to the manufacturers' instructions.

\section{Intracellular lactate dehydrogenase (LDH) activity measurement}

Cells were treated with metformin, cisplatin and drugloaded nano-cubosomal preparations for $48 \mathrm{~h}$ then collected and washed with PBS. Lactate dehydrogenase enzyme catalyzes the conversion of pyruvate, the end product of glycolysis, to lactate with the recycling of $\mathrm{NADH}$ back to $\mathrm{NAD}^{+}$. In this assay, the rate of decrease in NADH concentration is measured and is proportional to LDH concentration. NADH interacts with a specific probe to produce a color measured at $340 \mathrm{~nm}$.

\section{NADPH oxidase activity measurement}

Cells were grown in $75 \mathrm{~cm}^{2}$ flasks and allowed to adhere for $24 \mathrm{~h}$ then treated with the different treatment groups for $48 \mathrm{~h}$. Cells were then collected by trypsinisation and the cell pellet was washed twice with PBS. NADPH oxidase activity was measured using cytochrome-c reductase NADPH assay kit (Sigma, USA). The method depends on measurement of cytochrome-c reduction by $\mathrm{NADPH}$-cytochrome-c reductase in the presence of NADPH. The oxidation/reduction state of cytochrome-c alters the absorption spectrum. Cytochrome-c reduction is monitored by the increase in its absorbance at $550 \mathrm{~nm}$. 

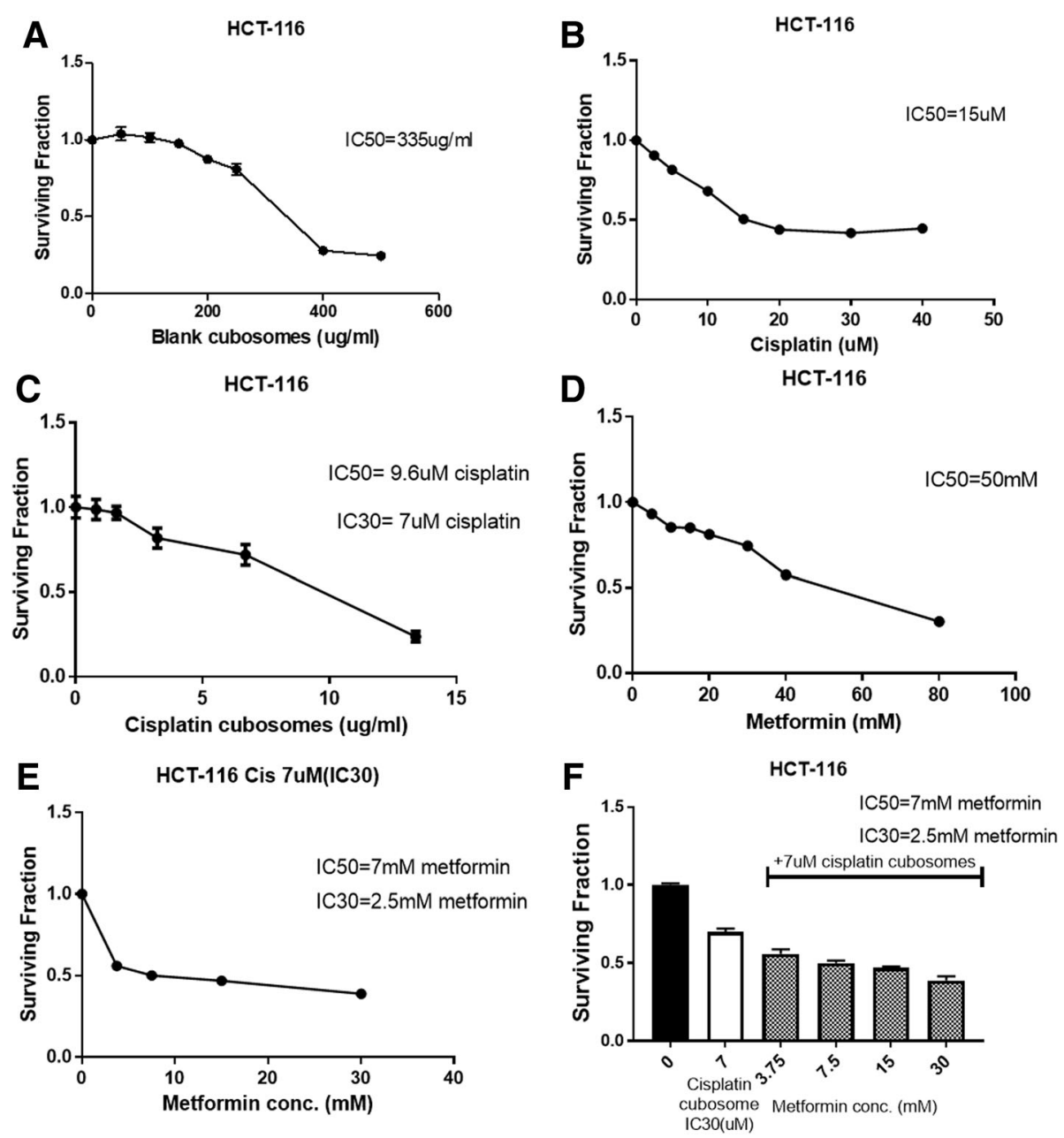

Fig. 3 Surviving fraction and the $\mathrm{IC}_{50}$ of HCT-116 cells after $48 \mathrm{~h}$ treatment with a blank nano-cubosomes $\mathbf{b}$ cisplatin $\mathbf{c}$ cisplatin nano-cubosomes d metformin e cisplatin-metformin nano-cubosomes $\mathbf{f} I C_{30}$ cisplatin nano-cubosomes and different metformin concentrations. The actual data represent the mean \pm SD of 3 separate experiments performed in sextuplets

Total AMPK, p002DAMPK, total mTOR, p-mTOR, total Akt and p-Akt protein level assessment

According to the kit manufacturer's instructions, total AMPK, p-AMPK alpha (S487), total mTOR, p-mTOR (S2448), total Akt and p-Akt (S473) protein levels were determined using RayBiotech Kits (Georgia, USA). Cell lysate samples were pipetted into the wells of a microplate pre-coated with polyclonal antibody against human AMPK, mTOR or Akt. The measured biomarkers present in the solutions were bound by the immobilized antibody and a color, measured at $450 \mathrm{~nm}$, is developed which is proportional to the amount of protein bound.

\section{Determination of Caspase-3 activity}

Samples of equal protein concentrations were assayed colorimetrically (Caspase-3/CPP32, BioVision, USA) according to the manufacturer's instructions to measure caspase- 3 activity. The assay depends on cleavage of the peptide from the color reporter molecule p-nitroaniline (pNA) by caspase-3 and chromophore detection at $405 \mathrm{~nm}$.

\section{Determination of protein content}

Protein amounts were measured by the Bradford method using Coomassie Protein Assay Kit (Pierce, USA) and all the results were expressed per mg protein content [10].

\section{Statistical analysis}

All values are expressed as mean \pm SD from a minimum of three different experiments. One way analysis of variance (ANOVA) was used followed by Tukey-Kramer multiple comparison test to determine the level of statistical significance which was considered at $p<0.05$. Statistical analysis was performed using Graphpad InStat, version 5.0 (Graphpad, USA). 

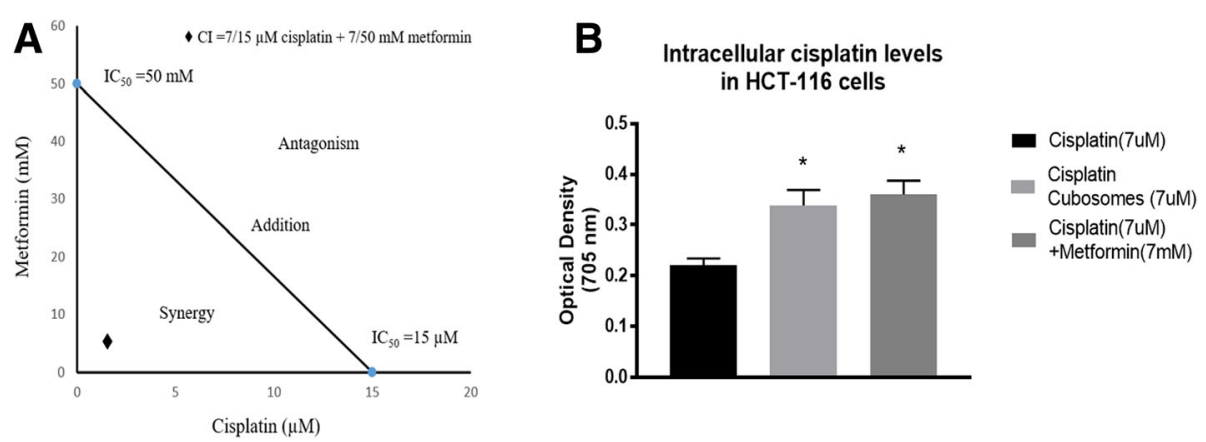

Fig. 4 a Combination index of metformin and cisplatin. b Optical density of cisplatin in HCT-116 cells treated with cisplatin (7 $\mu \mathrm{M})$, cisplatin nano-cubosomes $(7 \mu \mathrm{M})$ or cisplatin $(7 \mu \mathrm{M})$-metformin $(7 \mathrm{mM})$ nano-cubosome combination for $48 \mathrm{~h}$. All data are expressed as mean \pm SD of 3 separate experiments. The statistical significance of the results was analyzed using one way ANOVA followed by Tukey-Kramer multiple comparison test. ${ }^{*}$ Significantly different from cisplatin $(7 \mu \mathrm{M})(P<0.05)$

\section{Results}

Enhanced cisplatin cytotoxicity after nano-cubosomal incorporation with metformin

Treatment of HCT-116 with cisplatin resulted in an $\mathrm{IC}_{50}$ of $15 \mu \mathrm{M}$, while cisplatin-loaded nano-cubosomes led to a decrease in $\mathrm{IC}_{50}$ to $9.6 \mu \mathrm{M}$. Moreover, incorporation of $7 \mathrm{mM}$ metformin with $\mathrm{IC}_{30}$ cisplatin $(7 \mu \mathrm{M})$ in nano-cubosomes inhibited cell growth by $50 \%$. From the fraction of cell survival values, it is confirmed that the cytotoxic effects of the drug-loaded nanoparticles was higher than the effects of the individual drug (Fig. 3).

\section{Synergistic interaction between metformin and cisplatin in nano-cubosomes}

According to the isobologram equation used for determination of the type of drug interaction, cisplatin and metformin were found to have a synergistic effect with a combination index of 0.606 , less than 1 , as shown in (Fig. 4a).

\section{Increased cisplatin intracellular accumulation after incorporation into nano-cubosomes}

Measurement of intracellular cisplatin levels after $48 \mathrm{~h}$ of treatment with $7 \mu \mathrm{M}$ cisplatin, $7 \mu \mathrm{M}$ cisplatin nano-cubosomes and cisplatin (7 $\mu \mathrm{M})$-metformin (7 $\mathrm{mM}$ ) nano-cubosomes indicated that incorporation into nano-cubosomes increased drug uptake. This was evident in the nano-cubosomes treated cells where a 1.5-1.6 fold increase in cisplatin levels was observed compared to unformulated cisplatin (Fig. 4b).

\section{Depletion of glucose and ATP following nano-cubosomal treatment}

After $24 \mathrm{~h}$ of treatment a decrease in extracellular glucose level was detected in all treatment groups. Cisplatin nano-cubosomes and cisplatin-metformin nano-cubosomes showed the highest drop in glucose levels that reached 17 and $11 \mathrm{mg} / \mathrm{dl}$ compared to $42.5 \mathrm{mg} / \mathrm{dl}$ measured in the control group. An anticipated increase in the corresponding ATP levels was not observed. On the contrary all groups showed significant decrease in ATP which was prominent in the nano-cubosome containing groups.

Treatment of HCT-116 cells for $48 \mathrm{~h}$ with cisplatin resulted in ATP reduction in cell lysate by $18 \%$ accompanied by an increase in glucose uptake in an attempt to restore energy balance, leading to the decrease in its level in the medium by $60 \%$. On the other hand, cisplatin nano-cubosomes decreased ATP by $40 \%$ complemented with a greater diminution of glucose from the cell culture medium that reached $90 \%$. Addition of metformin to cisplatin nano-cubosomes resulted in an enhancement of the ATP reduction by $88 \%$ and a resultant glucose depletion reaching nearly undetectable levels (Fig. 5a and b).

\section{Increased AMP/ATP ratio in treated cells}

Figure $5 \mathrm{c}$ shows an increase in AMP/ATP ratio marking an increase in ATP consumption or decreased synthesis. Metformin, cisplatin, cisplatin nano-cubosomes and cisplatin-metformin nano-cubosomes showed an increase of 2.5, 1.7, 2.15 and 3.6 fold after $24 \mathrm{~h}$ of treatment compared to the control.

A marked elevation in the AMP/ATP ratio was detected after $48 \mathrm{~h}$ of treatment. It reached 3.6, 4.5, 6.5 and 8.8 fold in metformin, cisplatin, cisplatin nano-cubosomes and cisplatin-metformin nano-cubosomes groups.

\section{Initial activation followed by inhibition of intracellular LDH with a consequent rise in extracellular lactate upon nano-cubosomal treatment}

Figure 5d shows that treatment of HCT-116 cells with metformin or cisplatin for $24 \mathrm{~h}$ resulted in an increase in LDH activity of 1.15 and 1.25 folds respectively. Incorporation of cisplatin in nano-cubosomes increased LDH activity to 1.35 fold. Upon combination of both drugs, a 1.6 fold increase in the enzyme activity was 
observed that was statistically from all treatment groups. Coupled with the rise in enzyme activity an increase in extracellular lactate levels was detected with a similar pattern as shown in Fig. 5e.

After $48 \mathrm{~h}$ a shift in the enzyme activity was observed with a notable inhibition in all groups. Unformulated cisplatin decreased LDH activity by $17 \%$ while cisplatin nano-cubosomes decreased its activity by $33 \%$. Upon addition of metformin to cisplatin nano-cubosomes, LDH inhibition reached $70 \%$.

\section{Increased oxidative stress by cisplatin-metformin nano- cubosomes}

As a measure of oxidative stress, NADPH oxidase activity was assessed in all treatment groups. The drug alone produced a 2 fold increase in NADPH oxidase activity compared to a 2.8 fold increase produced by cisplatin nano-cubosomes. The combined formula produced a 3.4 fold rise in the enzyme activity that was statistically significant from the cisplatin group only (Fig. 5f).

\section{Dual inhibition of mTOR activity via pronounced AMPK activation and p-Akt suppression}

The nano-cubosomes containing both drugs produced a 7.5 fold increase in p-AMPK accompanied by a reduction in p-mTOR levels by $45 \%$. This was significantly different from the individual treatments, where metformin and cisplatin groups showed 4 and 2.25 fold increase compared to the control group. Cisplatin nano-cubosomes showed a 5.25 fold increase that was statistically significant from cisplatin only treatment. A consequent decrease in p-mTOR levels was noticed in all treatment groups with a similar pattern. Cisplatin treatment resulted in a $20 \%$ decrease, while metformin and cisplatin nano-cubosomes treatment groups produced a $35 \%$ decrease, respectively (Fig. 6).

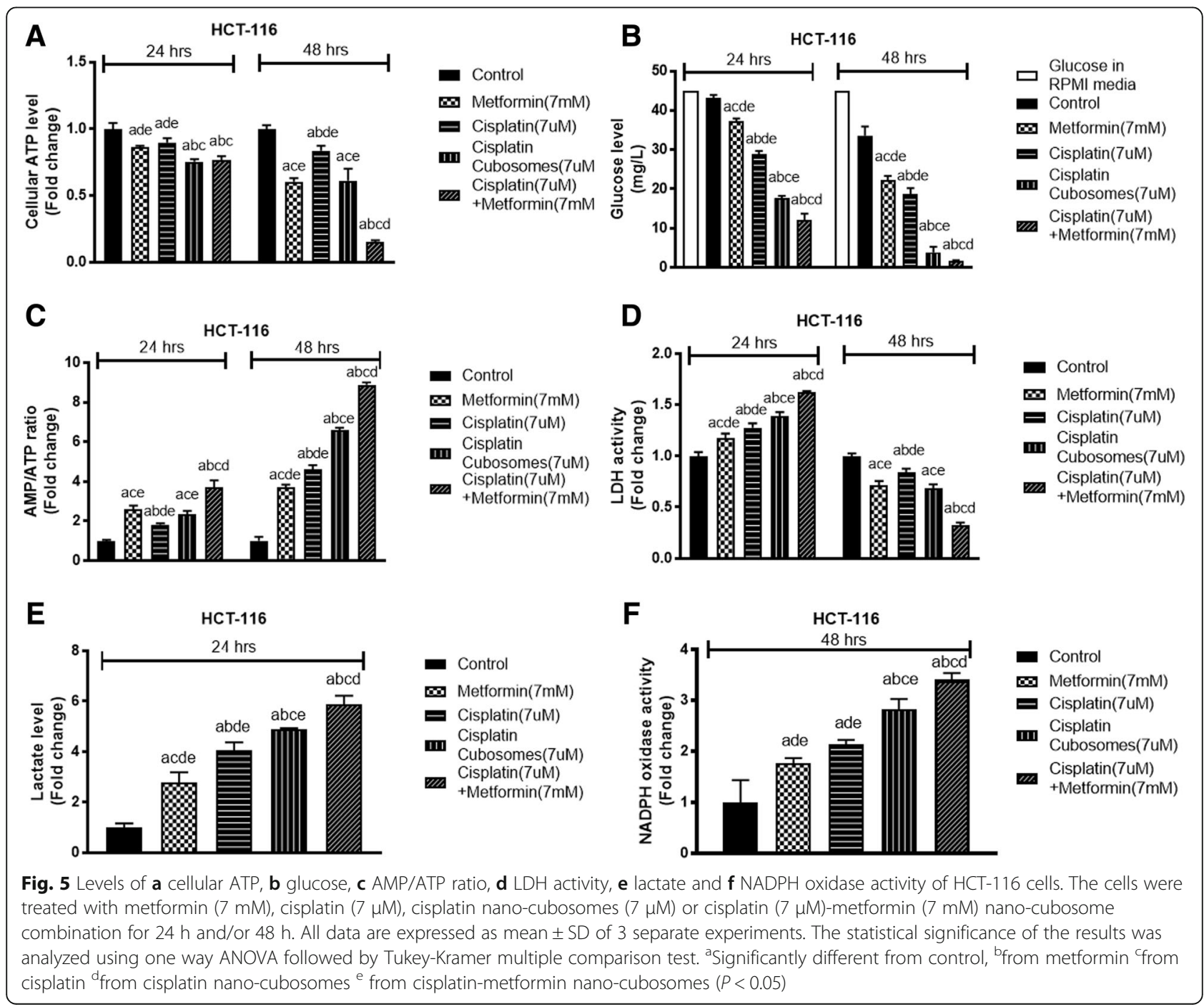




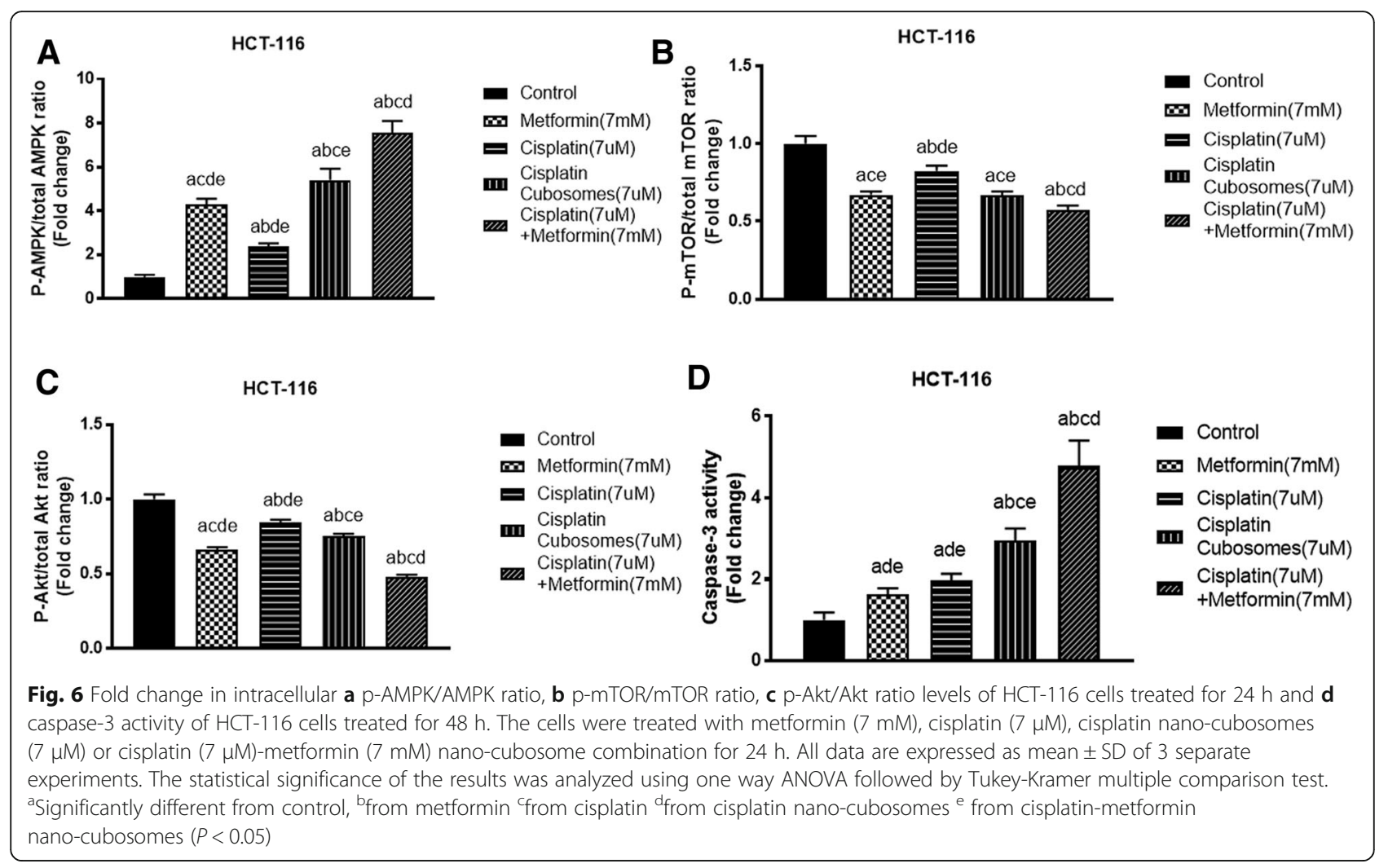

To further determine if mTOR was inhibited by other pathways we analyzed the levels of p-Akt. Metformin addition to the cisplatin nano-cubosomes reduced phosphorylated Akt levels by 55\% compared to the control. Cisplatin nano-cubosomes showed a robust inhibition of $28 \%$, while cisplatin alone had a lower effect evident as $18 \%$ decrease.

\section{Enhanced Caspase-3 activity}

As shown in Fig. 6d, administration of cisplatin and formulated cisplatin increased caspase- 3 activity two and three folds over the normal control. Incorporation of metformin into cisplatin nano-cubosomes produced an exaggerated 4.8 folds increase in caspase- 3 activity and apoptosis that was significant from all treatment groups.

\section{Discussion}

Drug-loaded lipid-based systems have become an outstanding theme of research in therapeutics [11, 12]. Amongst these, the monoolein-based nano-cubosomes emerged as one of the most cost-effective and clinically promising technology in disease diagnosis and treatment $[13,14]$. They possess a unique nanostructure consisting of a curved bilayer whose three-dimensional folding originates two disconnected, continuous water channels. This structure generates lipophilic and hydrophilic domains to integrate water-soluble, oil-soluble, and amphiphilic substances [15]. Apart from being biocompatible, biodegradable and lack of toxicity; it can incorporate drug amounts more than liposomes [16], as well as, protecting the drugs against physiological or chemical degradation [17].

Cisplatin is one of the platinum compounds used frequently in solid tumors. Due to the high rates of resistance in CRC, there were previous attempts to use cisplatin in the form of nanoparticles, showing that its efficacy and delivery to the tumor can be enhanced. However, little information is available on the cellular interaction of lipid-based cisplatin nanoparticles in vitro. In the present study, we show that cisplatin administration as nano-cubosomes alone or in combination with metformin demonstrated an exaggerated increase in CRC cell death using low drug concentrations.

One of the strategies to potentiate cisplatin cytotoxicity is glucose and ATP-deprivation either through inhibition of glycolytic or mitochondrial pathways [18]. Despite the fact that cisplatin decreases glucose transporter expression and thus glucose uptake and glycolysis, our results were in accordance with Liang et al. who stated that glucose uptake is increased in cisplatin sensitive cells [19]. Another study showed that under glucose-deprivation conditions, metformin enhanced cisplatin cytotoxicity in esophageal cancer cells [20]. Metformin targets cancer cells by various mechanisms, the mitochondria being its primary target 


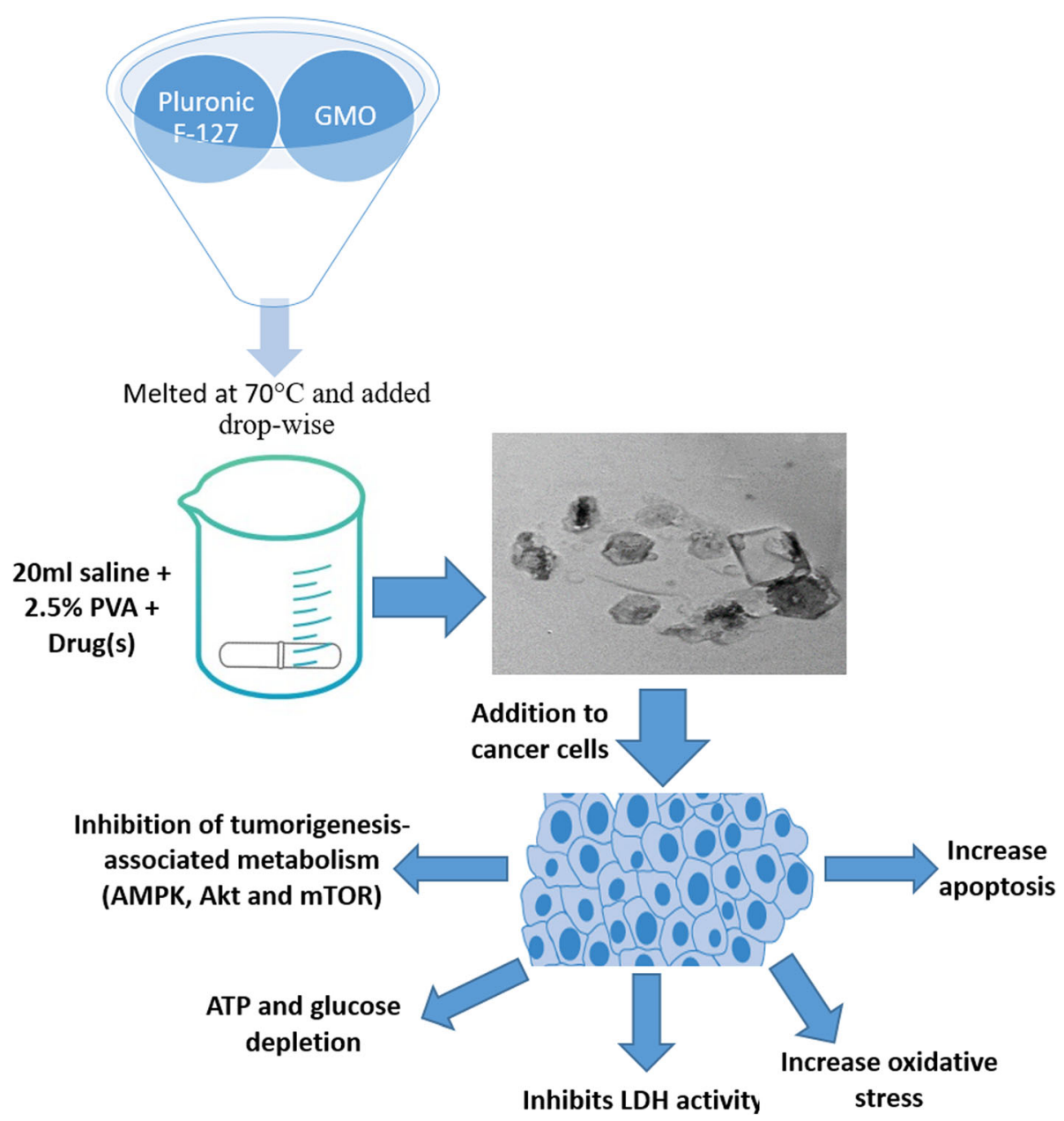

Fig. 7 Preparation of cisplatin and cisplatin-metformin nano-cubosomes using the emulsification technique. Treatment of CRC cells with drug-loaded nano-cubosomes result in a substantial inhibition of several metabolic pathways, including AMPK/mTOR and Akt/mTOR pathways. The resultant ATP and glucose depletion leads to an increased oxidative stress and therefore apoptosis. Another mechanism for the cytotoxic effect of the nano-cubosomes is the inhibition of LDH activity which in turn results in caspase-3 activation

where it leads to inhibition of several complexes and hence decreased ATP production. Activated AMPK, secondary to ATP depletion, inhibits mTOR and shuts down ATP-consuming pathways to maintain energy homeostasis under cellular stress conditions. It will therefore inhibit glucose, lipid and protein synthesis needed for cell growth, whereas fatty acid oxidation [21], glucose uptake and thus glycolysis are stimulated [22, 23]. Studies revealed the amplification of chemotherapy-induced AMPK activation by metformin followed by induction of tumor cell apoptosis [24]. The current study demonstrated a significant increase in AMPK levels, secondary to decreased ATP synthesis and/or increased utilization evident by the increase in AMP/ATP ratio, in nano-cubosomes-treated groups accompanied with increased glucose uptake (Fig. 7). This effect was profound in nano-cubosomes loaded with both drugs suggesting that metformin potentiates cisplatin effect.
mTOR is one of the important kinases that is deregulated in colon cancer [25]. Its activation results in cell growth, proliferation and survival. Several chemotherapy drugs target this kinase either directly or indirectly through activation/inhibition of its upstream signaling pathways including the LKB1/AMPK/mTOR and PI3K/ Akt/mTOR [26]. Experimental data showed that metformin significantly inhibited proliferation of chemo-resistant cells and its use as a neo-adjuvant chemotherapy improved patient response [24]. Apart from AMPK activation, metformin was previously reported to inhibit mTOR through Akt inactivation [27]. This dual inhibition of mTOR is advantageous since mTOR inhibitors, were reported to induce multiple resistance mechanisms, particularly feedback activation of Akt which displays abnormal signaling in colon cancer $[28,29]$. Here, we found that combined cisplatin-metformin nano-cubosomes significantly inhibited p-Akt and subsequent increase in mTOR 
levels despite Akt upregulation, normally found in CRC. This enhanced CRC cell sensitivity to mTOR inhibition is further indicative of an important role of mTOR in cancer cell proliferation and progression.

This study also demonstrated that the drug-loaded nano-cubosomes had an initial stimulatory followed by an inhibitory effect on LDH which is a cytosolic enzyme involved in glycolysis by catalyzing the inter-conversion of pyruvate and lactate. Increased activity of LDH may be due to the observed increase in the AMPK activity that subsequently increase glucose uptake and glycolysis. Several studies demonstrated an increase in glycolysis and LDH activity after metformin or cisplatin treatment due to inhibition of the mitochondrial oxidative phosphorylation [30, 31]. Nevertheless, LDH has multiple functions in neoplastic tissues. Aberrant LDH expression is common in several tumors, promoting reliance on glycolysis, generating lactate as an end-product [32] that enhances survival, metastasis and recurrence [33, 34]. LDH overexpression is accompanied with chemo-resistance, enhancement of angiogenesis and metastasis also through the elevation of vascular endothelial growth factor [35] and metalloproteinase levels [36]. The use of cisplatin-metformin nano-cubosomes demonstrated a significant inhibition of LDH activity after $48 \mathrm{~h}$ of treatment coupled with an increase in NADPH oxidase activity. This signals a rise in ROS production and hence increased apoptosis (Fig. 7).

\section{Conclusions}

In summary, cisplatin and cisplatin-metformin-loaded nano-cubosomes were successfully prepared by emulsification technique. They exhibited strong antitumor activity on human HCT-116 CRC cells in vitro compared to unformulated cisplatin. The nano-cubosomes affected several intracellular targets with significant inhibitory effect on tumorigenesis-associated metabolic pathways leading to increased apoptosis. Concisely, nano-cubosomes can be used as a potential carrier for enhancing cisplatin cytotoxicity. This cytotoxic effect can be further improved by the simultaneous presence of the indirect mTOR inhibitor, metformin, together with cisplatin in nano-cubosomal dispersions. Therefore, the prepared cisplatin and cisplatin-metformin nano-cubosomes could be potent agents in CRC treatment for further evaluation.

\footnotetext{
Abbreviations

Akt: Protein kinase B; AMPK: Adenosine monophosphate-activated protein kinase; CRC: Colorectal cancer; ELISA: Enzyme linked immunosorbent assay; GMO: Glyceryl monooleate; LDH: Lactate dehydrogenase; LKB1: Liver kinase B1; mTOR: Mammalian target of rapamycin; NADPH: Nicotinamide adenine dinucleotide phosphate hydrogen; PI3K: Phosphoinositide-3 kinase; PVA: Polyvinyl alcohol; ROS: Reactive oxygen species; SRB: Sulphorhodamine-B
}

\section{Funding}

This work did not receive any funding from the private or public sectors.
Availability of data and materials

The datasets generated and/or analyzed during the current study are available from the corresponding author on reasonable request.

\section{Authors' contributions}

The practical work and manuscript drafting was carried out by MS and AA. NN and SS participated in the design of the work, statistical analysis and manuscript revision. SS also offered tissue culture cancer lab. BS participated with the previous authors in designing and reviewing the manuscript. All authors read and approved the final manuscript.

Ethics approval and consent to participate

Not applicable

\section{Consent for publication \\ Not applicable}

\section{Competing interests}

The authors declare that they have no competing interests.

\section{Publisher's Note}

Springer Nature remains neutral with regard to jurisdictional claims in published maps and institutional affiliations.

\section{Author details}

${ }^{1}$ Department of Pharmacology and Toxicology, Faculty of Pharmacy, Cairo University, Kasr El-Aini St, Cairo 11562, Egypt. ${ }^{2}$ Institute of Molecular Medicine I, Medical Faculty, Heinrich-Heine-University, Universitätsstr. 1, Building 23.12, 40225 Düsseldorf, Germany. ${ }^{3}$ Department of Pharmaceutics and Industrial Pharmacy, Faculty of Pharmacy, Cairo University, Kasr El-Aini St, Cairo 11562, Egypt. ${ }^{4}$ Pharmacology Unit, Department of Cancer Biology, National Cancer Institute, Cairo University, Kasr El-Aini St., Fom El Khalig, Cairo 11796, Egypt.

Received: 6 March 2018 Accepted: 6 August 2018

Published online: 15 August 2018

\section{References}

1. Milczarek M, Rosinska S, Psurski M, Maciejewska M, Kutner A, Wietrzyk J. Combined colonic cancer treatment with vitamin D analogs and irinotecan or oxaliplatin. Anticancer Res. 2013;33(2):433-44.

2. Zhang ZJ, Zheng ZJ, Kan H, Song Y, Cui W, Zhao G, et al. Reduced risk of colorectal cancer with metformin therapy in patients with type 2 diabetes: a meta-analysis. Diabetes Care. 2011;34(10):2323-8.

3. Nangia-Makker P, Yu Y, Vasudevan A, Farhana L, Rajendra SG, Levi E, et al. Metformin: a potential therapeutic agent for recurrent colon cancer. PLoS One. 2014:9(1):e84369.

4. Pavlova NN, Thompson CB. The emerging hallmarks of Cancer metabolism. Cell Metab. 2016;23(1):27-47.

5. Duan X, He C, Kron SJ, Lin W. Nanoparticle formulations of cisplatin for cancer therapy. Wiley Interdiscip Rev Nanomed Nanobiotechnol. 2016 Sep; 8(5):776-91.

6. Al-Mahallawi AM, Abdelbary AA, Aburahma MH. Investigating the potential of employing bilosomes as a novel vesicular carrier for transdermal delivery of tenoxicam. Int J Pharm. 2015;485(1-2):329-40.

7. Morsi NM, Abdelbary GA, Ahmed MA. Silver sulfadiazine based cubosome hydrogels for topical treatment of burns: development and in vitro/in vivo characterization. Eur J Pharm Biopharm. 2014;86(2):178-89.

8. Thapa RK, Choi JY, Gupta B, Ramasamy T, Poudel BK, Ku SK, et al. Liquid crystalline nanoparticles encapsulating cisplatin and docetaxel combination for targeted therapy of breast cancer. Biomater Sci. 2016;4(9):1340-50.

9. Golla ED, Ayres GH. Spectrophotometric determination of platinum with o-phenylenediamine. Talanta. 1973;20(2):199-210.

10. Bradford MM. A rapid and sensitive method for the quantitation of microgram quantities of protein utilizing the principle of protein-dye binding. Anal Biochem. 1976;72:248-54.

11. Salunkhe SS, Bhatia NM, Bhatia MS. Implications of formulation design on lipid-based nanostructured carrier system for drug delivery to brain. Drug Deliv. 2016;23(4):1306-16.

12. Talluri SV, Kuppusamy G, Karri W, Tummala S, Madhunapantula SV. Lipidbased nanocarriers for breast cancer treatment - comprehensive review. Drug Deliv. 2016;23(4):1291-305. 
13. Caltagirone C, Falchi AM, Lampis S, Lippolis V, Meli V, Monduzzi M, et al. Cancer-cell-targeted theranostic cubosomes. Langmuir. 2014;30(21):6228-36.

14. Murgia S, Bonacchi S, Falchi AM, Lampis S, Lippolis V, Meli V, et al. Drug-loaded fluorescent cubosomes: versatile nanoparticles for potential theranostic applications. Langmuir. 2013;29(22):6673-9.

15. Rizwan SB, Assmus D, Boehnke A, Hanley T, Boyd BJ, Rades T, et al. Preparation of phytantriol cubosomes by solvent precursor dilution for the delivery of protein vaccines. Eur J Pharm Biopharm. 2011;79(1):15-22.

16. Siekmann B, Bunjes $\mathrm{H}$, Koch MH, Westesen K. Preparation and structural investigations of colloidal dispersions prepared from cubic monoglyceridewater phases. Int J Pharm. 2002;244(1-2):33-43.

17. Ganem-Quintanar A, Quintanar-Guerrero D, Buri P. Monoolein: a review of the pharmaceutical applications. Drug Dev Ind Pharm. 2000;26(8):809-20.

18. Pedersen PL. The cancer cell's "power plants" as promising therapeutic targets: an overview. J Bioenerg Biomembr. 2007;39(1):1-12.

19. Liang XJ, Finkel T, Shen DW, Yin JJ, Aszalos A, Gottesman MM. SIRT1 contributes in part to cisplatin resistance in cancer cells by altering mitochondrial metabolism. Mol Cancer Res. 2008;6(9):1499-506.

20. Yu H, Bian X, Gu D, He X. Metformin synergistically enhances cisplatin-induced cytotoxicity in esophageal squamous Cancer cells under glucose-deprivation conditions. Biomed Res Int. 2016;2016:8678634.

21. Merrill GF, Kurth EJ, Hardie DG, Winder WW. AICA riboside increases AMPactivated protein kinase, fatty acid oxidation, and glucose uptake in rat muscle. Am J Phys. 1997;273(6 Pt 1):E1107-12.

22. Barnes $\mathrm{K}$, Ingram JC, Porras OH, Barros LF, Hudson ER, Fryer LG, et al. Activation of GLUT1 by metabolic and osmotic stress: potential involvement of AMP-activated protein kinase (AMPK). J Cell Sci. 2002;115(Pt 11):2433-42.

23. Marsin AS, Bouzin C, Bertrand L, Hue L. The stimulation of glycolysis by hypoxia in activated monocytes is mediated by AMP-activated protein kinase and inducible 6-phosphofructo-2-kinase. J Biol Chem. 2002; 277(34):30778-83

24. Rocha GZ, Dias MM, Ropelle ER, Osorio-Costa F, Rossato FA, Vercesi AE, et al. Metformin amplifies chemotherapy-induced AMPK activation and antitumoral growth. Clin Cancer Res. 2011;17(12):3993-4005.

25. Slattery ML, Herrick JS, Lundgreen A, Fitzpatrick FA, Curtin K, Wolff RK. Genetic variation in a metabolic signaling pathway and colon and rectal cancer risk: mTOR, PTEN, STK11, RPKAA1, PRKAG2, TSC1, TSC2, PI3K and Akt1. Carcinogenesis. 2010;31(9):1604-11.

26. Wang XW, Zhang YJ. Targeting mTOR network in colorectal cancer therapy. World J Gastroenterol. 2014:20(15):4178-88.

27. Zhuang Y, Chan DK, Haugrud AB, Miskimins WK. Mechanisms by which low glucose enhances the cytotoxicity of metformin to cancer cells both in vitro and in vivo. PLoS One. 2014;9(9):e108444.

28. Awasthi N, Yen PL, Schwarz MA, Schwarz RE. The efficacy of a novel, dual PI3K mTOR inhibitor NVP-BEZ235 to enhance chemotherapy and antiangiogenic response in pancreatic cancer. J Cell Biochem. 2012;113(3):784-91.

29. Johnson SM, Gulhati P, Rampy BA, Han Y, Rychahou PG, Doan HQ, et al. Novel expression patterns of PI3K/Akt/mTOR signaling pathway components in colorectal cancer. J Am Coll Surg. 2010;210(5):767-8.

30. Viollet B, Guigas B, Sanz GN, Leclerc J, Foretz M, Andreelli F. Cellular and molecular mechanisms of metformin: an overview. Clin Sci (Lond). 2012; 122(6):253-70.

31. Choi YM, Kim HK, Shim W, Anwar MA, Kwon JW, Kwon HK, et al. Mechanism of cisplatin-induced cytotoxicity is correlated to impaired metabolism due to mitochondrial ROS generation. PLoS One. 2015;10(8):e0135083.

32. Liu X, Yang Z, Chen Z, Chen R, Zhao D, Zhou Y, et al. Effects of the suppression of lactate dehydrogenase a on the growth and invasion of human gastric cancer cells. Oncol Rep. 2015;33(1):157-62.

33. Walenta S, Wetterling M, Lehrke M, Schwickert G, Sundfor K, Rofstad EK, et al. High lactate levels predict likelihood of metastases, tumor recurrence, and restricted patient survival in human cervical cancers. Cancer Res. 2000;60(4):916-21.

34. Walenta S, Mueller-Klieser WF. Lactate: mirror and motor of tumor malignancy. Semin Radiat Oncol. 2004;14(3):267-74.

35. Koukourakis Ml, Giatromanolaki A, Sivridis E, Gatter KC, Harris AL. Lactate dehydrogenase 5 expression in operable colorectal cancer: strong association with survival and activated vascular endothelial growth factor pathway--a report of the tumour angiogenesis research group. J Clin Oncol. 2006;24(26):4301-8.

36. Baumann F, Leukel P, Doerfelt A, Beier CP, Dettmer K, Oefner PJ, et al. Lactate promotes glioma migration by TGF-beta2-dependent regulation of matrix metalloproteinase-2. Neuro-Oncology. 2009;11(4):368-80.

Ready to submit your research? Choose BMC and benefit from:

- fast, convenient online submission

- thorough peer review by experienced researchers in your field

- rapid publication on acceptance

- support for research data, including large and complex data types

- gold Open Access which fosters wider collaboration and increased citations

- maximum visibility for your research: over $100 \mathrm{M}$ website views per year

At BMC, research is always in progress.

Learn more biomedcentral.com/submissions 